\title{
Global AdS Picture of 1/2 BPS Wilson Loops
}

\author{
Kazumi Okuyama \\ Department of Physics, Shinshu University \\ Matsumoto 390-8621, Japan \\ kazumi@azusa.shinshu-u.ac.jp
}

\begin{abstract}
We study the holographic dual string configuration of $1 / 2$ BPS circular Wilson loops in $\mathcal{N}=4$ super Yang-Mills theory by using the global coordinate of AdS. The dual string worldsheet is given by the Poincaré disk $A d S_{2}$ sitting at a constant global time slice of $A d S_{5}$. We also analyze the correlator of two concentric circular Wilson loops from the global AdS perspective and study the phase transition associated with the instability of annulus worldsheet connecting the two Wilson loops.
\end{abstract}

December 2009 


\section{Introduction}

In the AdS/CFT correspondence between $\mathcal{N}=4$ super Yang-Mills (SYM) and the type IIB string theory on $A d S_{5} \times S^{5}$, supersymmetric Wilson loops are interesting objects to study. On the bulk gravity side, the expectation value of such Wilson loops is obtained by computing the minimal area of string worldsheet ending on the loop at the boundary of AdS [1,2]. The string worldsheet which is holographically dual to a supersymmetric Wilson loop is generally characterized as a pseudo-holomorphic curve on $A d S_{5} \times S^{5}$ [3, 1 , However, it would be nice to have a more intuitive picture of dual worldsheet.

In the case of a 1/2 BPS circular Wilson loop, the dual worldsheet was obtained in the Pincaré coordinate of $A d S_{5}$ [5]. In this paper, we consider the worldsheet dual of $1 / 2$ BPS circular Wilson loops using the global coordinate of $A d S_{5}$. This global AdS description of Wilson loop is closely related to the radial quantization of $\mathcal{N}=4 \mathrm{SYM}$, since the $\mathcal{N}=4$ SYM defined on $\mathbb{R} \times S^{3}$ is dual to type IIB string theory on global $A d S_{5}$. We find that the dual worldsheet of $1 / 2$ BPS circular Wilson loop is the Poincaré disk sitting at a fixed

global time of $A d S_{5}$. Using this picture, we revisit the holographic computation of the correlator of two concentric 1/2 BPS circular Wilson loops studied in [6]. As discussed in [6], when the ratio of radii of the two circles exceeds a certain critical value, the worldsheet of annulus topology in the bulk AdS ceases to exist, and this leads to an analogue of Gross-Ooguri phase transition [7]. We analyze the annulus worldsheet connecting the two loops using the global coordinate of $A d S_{5}$.

This paper is organized as follows. In section 2, we first consider the radial quantization picture of 1/2 BPS circular Wilson loops in the SYM side. Next we study the dual worldsheet ending on the circular loop using the global coordinate of $A d S_{5}$. In section 3 , we study the correlator of two concentric circular Wilson loops from the global AdS perspective and consider the Gross-Ooguri type transition of this correlator. In section 4, we discuss some interesting future directions.

\section{1/2 BPS Wilson Loops from Global AdS Perspective}

\subsection{Circular Wilson Loops in Radial Quantization}

In this paper we consider Wilson loops in $\mathcal{N}=4 \mathrm{SYM}$ on Euclidean signature space. It is well-known that the $1 / 2$ BPS Wilson loop in $\mathcal{N}=4$ SYM is given by [1]

$$
W(C)=\frac{1}{N} \operatorname{Tr} P \exp \left[\oint_{C} d s\left(i A_{\mu}(x) \dot{x}^{\mu}(s)+\theta^{I} \Phi_{I}(x)|\dot{x}(s)|\right)\right],
$$


where $C$ is a circle or a straight line on $\mathbb{R}^{4}$ in order to preserve $1 / 2$ of supersymmetry. $\theta^{I}$ in (2.1) is a constant unit 6-vector which specifies a point on $S^{5}$. Let us consider a circular Wilson loop $C$ with radius $a$

$$
C: \quad x_{1}^{2}+x_{2}^{2}=a^{2}, \quad x_{3}=x_{4}=0 .
$$

Here $x_{\mu}(\mu=1, . ., 4)$ denote the coordinate of Euclidean $\mathbb{R}^{4}$.

In the radial quantization with respect to the origin of $\mathbb{R}^{4}$, we introduce the radial time $\tau$ as the $\log$ of radial coordinate $r=\sqrt{x_{\mu}^{2}}$. Then the coordinate $x_{\mu}$ of $\mathbb{R}^{4}$ is written as

$$
x_{\mu}=e^{\tau} n_{\mu},
$$

where $n_{\mu}$ is a unit 4-vector $n_{\mu}^{2}=1$ parametrizing $S^{3}$. By the change of variable $r=e^{\tau}$, the metric of $\mathbb{R}^{4}$ becomes conformally equivalent to the metric of $\mathbb{R} \times S^{3}$

$$
d s^{2}=d r^{2}+r^{2} d \Omega_{3}^{2}=e^{2 \tau}\left(d \tau^{2}+d \Omega_{3}^{2}\right),
$$

where $d \Omega_{3}^{2}$ is the metric of unit 3-sphere. In this radial quantization picture, the circular loop $C$ in (2.2) becomes a great circle of $S^{3}=\left\{n_{\mu}^{2}=1\right\}$, and $C$ is sitting at the constant radial time $\tau$

$$
\tau=\log a, \quad n_{1}^{2}+n_{2}^{2}=1
$$

In the radial quantization, a local operator $\mathcal{O}_{\Delta}$ inserted at the origin of $\mathbb{R}^{4}$ corresponds to a state $|\Delta\rangle$ via the state-operator correspondence

$$
|\Delta\rangle=\mathcal{O}_{\Delta}(0)|0\rangle
$$

What does the circular Wilson loop centered at the origin of $\mathbb{R}^{4}$ correspond to in the radial quantization? The above argument suggests that the circular Wilson loop $W(C)$ with radius $a$ corresponds to an operator $\widehat{W}_{C}(\tau)$ inserted on a fixed time slice $\tau=\log a$ of $\mathbb{R} \times S^{3}$, and this operator $\widehat{W}_{C}(\tau)$ acts on the Hilbert space $\mathcal{H}=\{|\Delta\rangle\}$ of $\mathcal{N}=4$ SYM. For instance, the OPE of a Wilson loop $W(C)$ and a local operator $\mathcal{O}_{\Delta}$ is written as

$$
\left\langle W(C) \mathcal{O}_{\Delta}(0)\right\rangle=\left\langle 0\left|\widehat{W}_{C}(\tau)\right| \Delta\right\rangle
$$

Similarly, the correlator of $n$ concentric 1/2 BPS circular Wilson loops is written as

$$
\left\langle W\left(C_{1}\right) \cdots W\left(C_{n}\right)\right\rangle=\left\langle 0\left|\mathbf{T}\left[\widehat{W}_{C_{1}}\left(\tau_{1}\right) \cdots \widehat{W}_{C_{n}}\left(\tau_{n}\right)\right]\right| 0\right\rangle,
$$

where $\mathbf{T}$ denotes the time ordering with respect to the radial time $\tau$ (or the radial ordering in the original $\mathbb{R}^{4}$ picture) and $\tau_{i}(i=1, . ., n)$ is related to the radius $a_{i}$ of loop $C_{i}$ by $\tau_{i}=\log a_{i}$ 


\subsection{Holographic Dual of Circular Wilson Loops}

The gravity dual of Wilson loop in $\mathcal{N}=4 \mathrm{SYM}$ is given by the string worldsheet in $A d S_{5} \times S^{5}$ bounded by the loop at the boundary of $A d S_{5}$ [1],2]. In the case of $1 / 2$ BPS circular Wilson loop, the dual string worldsheet was obtained as a minimal surface in $A_{d} S_{5}$ which minimizes the Nambu-Goto action. In the Poincaré coordinate of (Euclidean) $A d S_{5}$

$$
d s_{A d S_{5}}^{2}=\frac{d z^{2}+d x_{\mu} d x^{\mu}}{z^{2}}
$$

the dual string worldsheet of circular Wilson loop $C$ (2.2) is given by [5]

$$
\Sigma: \quad z^{2}+x_{1}^{2}+x_{2}^{2}=a^{2}, \quad x_{3}=x_{4}=0 .
$$

One can easily see that this surface $\Sigma$ ends on the loop $C(2.2)$ at the boundary $z=0$ of $A d S_{5}$.

What does this surface $\Sigma$ look like in the global coordinate of $A d S_{5}$ ? The metric of $A d S_{5}$ in the global coordinate is given by

$$
d s_{A d S_{5}}^{2}=\cosh ^{2} \rho d \tau^{2}+d \rho^{2}+\sinh ^{2} \rho d \Omega_{3}^{2}
$$

To see the relation between the Poincaré coordinate and the global coordinate, it is convenient to view $A d S_{5}$ as a hypersurface in $\mathbb{R}^{1,5}$

$$
\eta_{a b} Y^{a} Y^{b}=-1
$$

where $Y^{a}(a=0, \ldots, 5)$ denotes the coordinate of $\mathbb{R}^{1,5}$ with metric $\eta_{a b}=(-+++++)$. Above, we have set the $\operatorname{AdS}$ radius $R_{A d S}=1$ for simplicity. In terms of the Poincaré coordinate $\left(z, x^{\mu}\right), Y^{a}$ is given by

$$
Y^{0}+Y^{5}=z+\frac{x_{\mu}^{2}}{z}, \quad Y^{0}-Y^{5}=\frac{1}{z}, \quad Y^{\mu}=\frac{x^{\mu}}{z} \quad(\mu=1, \ldots, 4)
$$

On the other hand, $Y^{a}$ is written in the global coordinate as

$$
Y^{0} \pm Y^{5}=e^{ \pm \tau} \cosh \rho, \quad Y^{\mu}=n^{\mu} \sinh \rho,
$$

where $n^{\mu}$ is a unit 4-vector $n_{\mu}^{2}=1$ parametrizing $S^{3}$. From (2.13) and (2.14), the Poincaré coordinate $\left(x, x^{\mu}\right)$ and the global coordinate $\left(\tau, \rho, n^{\mu}\right)$ are related as

$$
z=\frac{e^{\tau}}{\cosh \rho}, \quad x^{\mu}=e^{\tau} n^{\mu} \tanh \rho .
$$


One can check that the metric of $A d S_{5}$ in Poincaré coordinate (2.9) becomes the metric in the global coordinate (2.11) under this change of variables (2.15).

In the global coordinate, the boundary of $A d S_{5}$ is located at $\rho=\infty$. At the boundary of $A d S_{5},\left(z, x^{\mu}\right)$ in $(2.15)$ becomes

$$
z \rightarrow 0, \quad x^{\mu} \rightarrow e^{\tau} n^{\mu} \quad(\rho \rightarrow \infty)
$$

By comparing (2.3) and (2.16), we find that the radial time $\tau$ of $\mathbb{R} \times S^{3}$ is identified with the global time $\tau$ of $A d S_{5}$ at the boundary $\rho=\infty$. This is consistent with the fact that the $\mathcal{N}=4 \mathrm{SYM}$ defined on $\mathbb{R} \times S^{3}$ is dual to the type IIB string theory on global $A d S_{5}$.

Now let us rewrite the minimal surface $\Sigma(2.10)$ using the global coordinate. It turns out that the equation of $\Sigma$ in the global coordinate is simply given by the same equation as the circular loop $C$ in the radial quantization picture (2.5)

$$
\tau=\log a, \quad n_{1}^{2}+n_{2}^{2}=1
$$

For this configuration of worldsheet $\Sigma$, the unit 4 -vector $n^{\mu}$ in (2.14) can be taken as

$$
\left(n_{1}, n_{2}, n_{3}, n_{4}\right)=(\cos \phi, \sin \phi, 0,0)
$$

Plugging (2.17) and (2.18) into the relation between Poincaré and global coordinates (2.15), we get

$$
z=\frac{a}{\cosh \rho}, \quad x_{1}+i x_{2}=a e^{i \phi} \tanh \rho
$$

We can easily see that $\left(z, x_{1}, x_{2}\right)$ in $(2.19)$ satisfy the equation of $\Sigma$ in the Poincaé coordinate (2.10). Therefore, we conclude that (2.17) is the equation of minimal surface $\Sigma$ written in the global coordinate. We can also show that the metric on $\Sigma$ induced from the global $A d S_{5}$ metric (2.11) is the metric of $A d S_{2}$

$$
d s_{\Sigma}^{2}=d \rho^{2}+\sinh ^{2} \rho d \phi^{2}
$$

To summarize, the minimal surface $\Sigma$ in the global coordinate is a Poincaré disk $A d S_{2}$ parametrized by $\rho \in[0, \infty]$ and $\phi \in[0,2 \pi]$ and $\Sigma$ is sitting at a constant time slice $\tau=\log a$ of global $A d S_{5}$ (see Fig. 1). Note that $\phi$ in (2.18) is the angle coordinate of the great circle $S^{1}=\left\{n_{1}^{2}+n_{2}^{2}=1\right\}$ on the 3 -sphere $S^{3}=\left\{n_{\mu}^{2}=1\right\}$. 


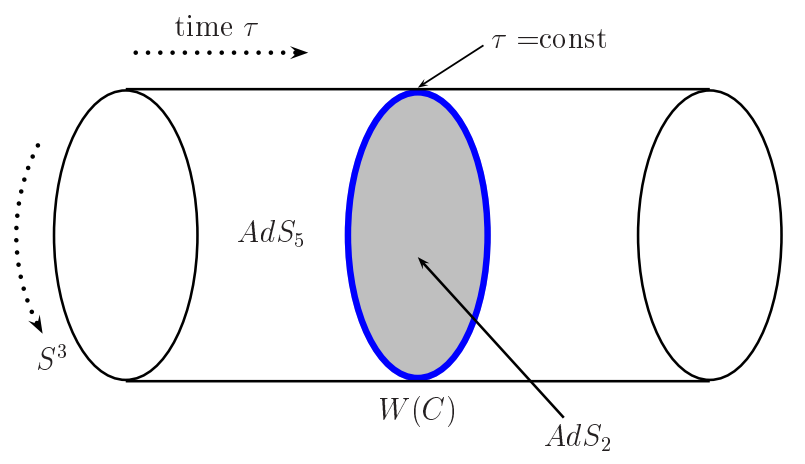

Fig. 1: The string worldsheet $\Sigma$ dual to a $1 / 2$ BPS circular Wilson loop $W(C)$ is the Poincaré disk $A d S_{2}$ bounded by the loop $C . \Sigma$ is sitting at a constant time slice of global $A d S_{5}$.

\subsection{Nambu-Goto Action in Global Coordinate}

In this subsection we consider the equation of motion of Nambu-Goto string using the global coordinate. The Nambu-Goto action in $A d S_{5}$ is given by

$$
S=\frac{\sqrt{\lambda}}{2 \pi} \int d^{2} \sigma \sqrt{\operatorname{det} G},
$$

where $G$ is the worldsheet metric induced from the global $A d S_{5}$ (2.11). Here we used the fact that the string tension in the unit of AdS radius is related to the 't Hooft coupling $\lambda=g_{\mathrm{YM}}^{2} N$ of $\mathcal{N}=4 \mathrm{SYM}$ as

$$
T_{F 1} R_{A d S}^{2}=\frac{R_{A d S}^{2}}{2 \pi \alpha^{\prime}}=\frac{\sqrt{\lambda}}{2 \pi} .
$$

In order to analyze the configuration of string worldsheet in the global coordinate of $A d S_{5}$ (2.11), we make an ansatz

$$
\rho=\sigma_{0}, \quad \phi=\sigma_{1}, \quad \tau=\tau\left(\sigma_{0}\right)=\tau(\rho)
$$

where $\left(\sigma_{0}, \sigma_{1}\right)$ is the two-dimensional worldsheet coordinate. As in the previous subsection, $\phi$ denotes the angular coordinate of a great circle $S^{1} \subset S^{3}$ inside $A d S_{5}$. For this configuration (2.23), the worldsheet metric induced from $A d S_{5}$ (2.11) is given by

$$
d s_{F 1}^{2}=\left[\cosh ^{2} \rho\left(\frac{d \tau}{d \rho}\right)^{2}+1\right] d \rho^{2}+\sinh ^{2} \rho d \phi^{2}
$$

and the Nambu-Goto action (2.21) becomes

$$
S=\frac{\sqrt{\lambda}}{2 \pi} \int d \phi d \rho \sinh \rho \sqrt{\cosh ^{2} \rho\left(\frac{d \tau}{d \rho}\right)^{2}+1} .
$$


The equation of motion following from this action is

$$
\frac{d}{d \rho}\left(\frac{\sinh \rho \cosh ^{2} \rho \frac{d \tau}{d \rho}}{\sqrt{\cosh ^{2} \rho\left(\frac{d \tau}{d \rho}\right)^{2}+1}}\right)=0 .
$$

Clearly, $\frac{d \tau}{d \rho}=0$ is a solution of this equation and the induced metric (2.24) for the $\frac{d \tau}{d \rho}=0$ case is

$$
d s_{F 1}^{2}=d \rho^{2}+\sinh ^{2} \rho d \phi^{2}
$$

This is nothing but the metric of $A d S_{2}$. This analysis gives a direct check that the Poincaré disk sitting at $\tau=\log a$ is a minimal surface.

The on-shell action of the minimal surface has a divergence coming from the large $\rho$ region, and we need to regularize it by introducing the IR cut-off $\rho_{0}$ in the $\rho$-integral (2.25)

$$
S=\frac{\sqrt{\lambda}}{2 \pi} \int_{0}^{2 \pi} d \phi \int_{0}^{\rho_{0}} d \rho \sinh \rho=\sqrt{\lambda}\left(\cosh \rho_{0}-1\right) .
$$

The first term $\sqrt{\lambda} \cosh \rho_{0}$ in 2.28 diverges in the limit $\rho_{0} \rightarrow \infty$, and this can be removed by adding a boundary term to the action日 [1,9]. After removing this term, we get the regularized action

$$
S_{\mathrm{reg}}=-\sqrt{\lambda}
$$

which gives the leading large $\lambda$ behavior of Wilson loop expectation value

$$
\langle W(C)\rangle \approx e^{-S_{\mathrm{reg}}}=e^{\sqrt{\lambda}}
$$

This large $\lambda$ behavior of $\langle W(C)\rangle$ is reproduced by the exact computation in the $\mathcal{N}=4$ SYM side using the localization of path integral to a Gaussian matrix model [10, 11, 12].

\section{Two Concentric Circular Wilson Loops}

In this section, we study the correlator of two concentric circular Wilson loops from the holographic dual viewpoint. This problem is studied in 6$]^{2}$ and it is found that as

1 This procedure is reminiscent of the quantum entropy function formalism of Sen [8].

2 The holographic dual of two Wilson loops with equal radii separated in the $x_{3}$-direction was studied in 13, 14. 
we vary the ratio $\frac{a_{2}}{a_{1}}$ of the radii $a_{1}, a_{2}$ of two circles there is a phase transition similar to that found by Gross and Ooguri [7]. We revisit this problem using the global coordinate of $A d S_{5}$. As we will see below, the use of global coordinate enables us to visualize the dual worldsheet very clearly.

In the radial quantization picture, the correlator $\left\langle W\left(C_{1}\right) W\left(C_{2}\right)\right\rangle$ of two circular Wilson loops is written as the two operator insertions at $\tau_{i}=\log a_{i}(i=1,2)$

$$
\left\langle W\left(C_{1}\right) W\left(C_{2}\right)\right\rangle=\left\langle 0\left|\widehat{W}_{C_{2}}\left(\tau_{2}\right) \widehat{W}_{C_{1}}\left(\tau_{1}\right)\right| 0\right\rangle .
$$

Here we have assumed $\tau_{2}>\tau_{1}$ without loss of generality. The two Wilson loops are separated in the $\tau$-direction by the amount $\tau_{0}$

$$
\tau_{0}=\tau_{2}-\tau_{1}=\log \frac{a_{2}}{a_{1}}
$$

This correlator has a connected part and a disconnected part

$$
\left\langle W\left(C_{1}\right) W\left(C_{2}\right)\right\rangle=\left\langle W\left(C_{1}\right) W\left(C_{2}\right)\right\rangle_{\mathrm{conn}}+\left\langle W\left(C_{1}\right)\right\rangle\left\langle W\left(C_{2}\right)\right\rangle .
$$

(A)

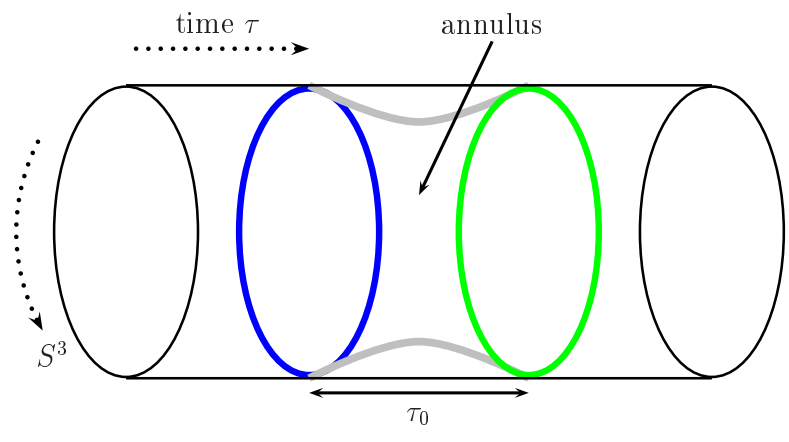

(B)

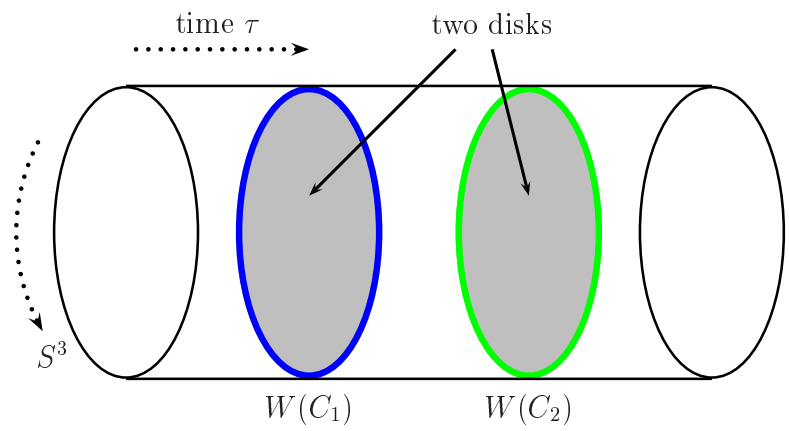

Fig. 2: (A) The worldsheet of annulus topology connecting the two loops $C_{1}, C_{2}$ contributes to the connected part of the correlator of two Wilson loops $\left\langle W\left(C_{1}\right) W\left(C_{2}\right)\right\rangle_{\text {conn }}$. The minimal surface of annulus topology does not exist if $\tau_{0}$ is larger than some critical value $\tau_{c}$. (B) The two disconnected disk worldsheets correspond to the disconnected part of Wilson loop correlator $\left\langle W\left(C_{1}\right)\right\rangle\left\langle W\left(C_{2}\right)\right\rangle$. 
In the large 't Hooft coupling regime, the correlator $\left\langle W\left(C_{1}\right) W\left(C_{2}\right)\right\rangle$ has a holographic dual description as a string worldsheet having the two circles $C_{1}$ and $C_{2}$ as boundaries. In the global $A d S_{5}$ picture, the disconnected part corresponds to the two Poincaré disks sitting at $\tau=\tau_{1}$ and $\tau=\tau_{2}$, and the connected part corresponds to the annulus worldsheet connecting $C_{1}$ and $C_{2}$ (see Fig. 2). Then the two-loop correlator (3.3) in the large $\lambda$ regime is approximately given by the regularized Nambu-Goto action

$$
\begin{aligned}
& \left\langle W\left(C_{1}\right) W\left(C_{2}\right)\right\rangle_{\mathrm{conn}} \approx e^{-S_{\text {reg }}(\text { annulus })}, \\
& \left\langle W\left(C_{1}\right)\right\rangle\left\langle W\left(C_{2}\right)\right\rangle \approx e^{-2 S_{\text {reg }}(\text { disk })}=e^{2 \sqrt{\lambda}} .
\end{aligned}
$$

In the following we study the annulus worldsheet connecting the two circles using the global $A d S_{5}$ coordinate. We make the same ansatz (2.23) for the string configuration as in the disk case, hence the equation of motion we should solve is (2.26). This equation (2.26) can be integrated once

$$
\frac{\sinh \rho \cosh ^{2} \rho \frac{d \tau}{d \rho}}{\sqrt{\cosh ^{2} \rho\left(\frac{d \tau}{d \rho}\right)^{2}+1}}=\text { const. }
$$

Using the translation invariance in the $\tau$-direction, we can set the locations of two loops to be at $\tau_{1}=-\frac{1}{2} \tau_{0}$ and $\tau_{2}=\frac{1}{2} \tau_{0}$. In order for the annulus worldsheet to end on the two circles at the boundary of $A d S_{5}, \tau(\rho)$ should satisfy the following boundary condition

$$
\lim _{\rho \rightarrow \infty} \tau(\rho)= \pm \frac{\tau_{0}}{2}
$$

Also, we require that $\tau(\rho)$ is vertical at the turning point $\rho=\rho_{\min }$ (see Fig. 2(A))

$$
\lim _{\rho \rightarrow \rho_{\min }} \frac{d \tau}{d \rho}=\infty
$$

From this condition the constant on the right hand side of (3.5) is fixed as

$$
\frac{\sinh \rho \cosh ^{2} \rho \frac{d \tau}{d \rho}}{\sqrt{\cosh ^{2} \rho\left(\frac{d \tau}{d \rho}\right)^{2}+1}}=\cosh \rho_{\min } \sinh \rho_{\min } .
$$

From this equation we get

$$
\frac{d \tau}{d \rho}= \pm \frac{\sinh ^{2} 2 \rho_{\min }}{\cosh \rho \sqrt{\sinh ^{2} 2 \rho-\sinh ^{2} 2 \rho_{\min }}} .
$$


The plus sign of (3.9) represents the right half of annulus $0 \leq \tau \leq \frac{1}{2} \tau_{0}$, and the minus sign corresponds to the left half $-\frac{1}{2} \tau_{0} \leq \tau \leq 0$ (see Fig. 2(A)). On the right half of annulus, the equation (3.9) is solved as

$$
\tau(\rho)=\int_{\rho_{\min }}^{\rho} \frac{d \rho}{\cosh \rho} \frac{\sinh 2 \rho_{\min }}{\sqrt{\sinh ^{2} 2 \rho-\sinh ^{2} 2 \rho_{\min }}} .
$$

The boundary condition (3.6) leads to the relation between $\tau_{0}$ and $\rho_{\text {min }}$

$$
\tau_{0}=2 \int_{\rho_{\min }}^{\infty} \frac{d \rho}{\cosh \rho} \frac{\sinh 2 \rho_{\min }}{\sqrt{\sinh ^{2} 2 \rho-\sinh ^{2} 2 \rho_{\min }}} \equiv f\left(\rho_{\min }\right) .
$$

By performing the change of variable $t=\frac{\sinh \rho_{\min }}{\sinh \rho}$, the function $f\left(\rho_{\min }\right)$ in (3.11) is written as 3

$$
f\left(\rho_{\min }\right)=2 \cosh \rho_{\min }\left[K\left(i \operatorname{coth} \rho_{\min }\right)-\Pi\left(-\frac{1}{\sinh ^{2} \rho_{\min }}, i \operatorname{coth} \rho_{\min }\right)\right],
$$

where $K(k)$ and $\Pi(n, k)$ denote the elliptic integrals of the first and third kind, respectively

$$
K(k)=\int_{0}^{1} \frac{d t}{\sqrt{\left(1-t^{2}\right)\left(1-k^{2} t^{2}\right)}}, \quad \Pi(n, k)=\int_{0}^{1} \frac{d t}{\left(1-n t^{2}\right) \sqrt{\left(1-t^{2}\right)\left(1-k^{2} t^{2}\right)}} .
$$

The function $f\left(\rho_{\text {min }}\right)$ vanishes in the limit $\rho_{\min } \rightarrow 0, \infty$ as

$$
\begin{aligned}
& f\left(\rho_{\text {min }}\right) \sim-2 \rho_{\text {min }} \log \rho_{\text {min }} \quad\left(\rho_{\text {min }} \rightarrow 0\right), \\
& f\left(\rho_{\text {min }}\right) \sim \frac{4 \sqrt{2 \pi^{3}}}{\Gamma\left(\frac{1}{4}\right)^{2}} e^{-\rho_{\text {min }}} \quad\left(\rho_{\text {min }} \rightarrow \infty\right),
\end{aligned}
$$

and $f\left(\rho_{\min }\right)$ has a single maximum at some finite $\rho_{\min }=\rho_{c}$ (see Fig. 3). As discussed in [6], the existence of a maximal value $\tau_{c}=f\left(\rho_{c}\right)$ of the function $f\left(\rho_{\text {min }}\right)$ means that the annulus worldsheet ceases to exist when the separation $\tau_{0}$ between the two loops is larger than $\tau_{c}$, and the annulus configuration exists only when $0 \leq \tau_{0} \leq \tau_{c}$. This is analogous to the phase transition discussed by Gross and Ooguri [7].

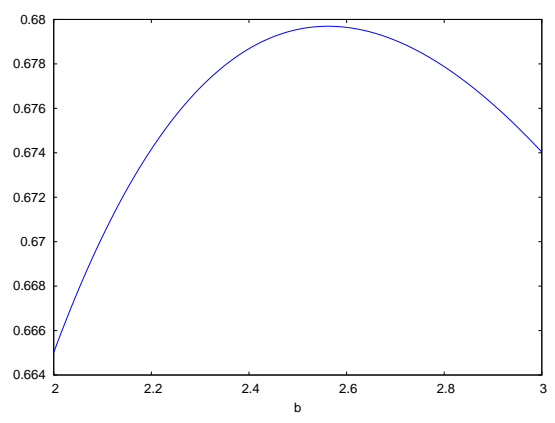

Fig. 3: This is a plot of $f\left(\rho_{\min }\right)$ as a function of $b=\frac{1}{\sinh \rho_{\min }}$. The function $f\left(\rho_{\text {min }}\right)$ has a single maximum with maximal value $\tau_{c}(\sim 0.68)$.

3 Our function $f\left(\rho_{\min }\right)$ corresponds to the function $2 F(k a)$ in [13,6]. 
Next we consider the on-shell action for this annulus configuration. Plugging $\frac{d \tau}{d \rho}$ in (3.9) into the action (2.25), we find

$$
\begin{aligned}
S & =2 \sqrt{\lambda} \int_{\rho_{\text {min }}}^{\infty} d \rho \sinh \rho \sqrt{\cosh ^{2} \rho\left(\frac{d \tau}{d \rho}\right)^{2}+1} \\
& =\sqrt{\lambda} \int_{\rho_{\min }}^{\infty} \frac{d \rho}{\cosh \rho} \frac{\sinh ^{2} 2 \rho}{\sqrt{\sinh ^{2} 2 \rho-\sinh ^{2} 2 \rho_{\min }}}
\end{aligned}
$$

The factor of 2 in the first line of (3.15) comes from the fact that there are two branches $( \pm$ in $(3.9))$ for $\tau(\rho)$. To evaluate this integral, we separate it into two parts

$$
\begin{aligned}
S & =S^{(1)}+S^{(2)}, \\
S^{(1)} & =\sqrt{\lambda} \int_{\rho_{\min }}^{\infty} \frac{d \rho}{\cosh \rho} \sqrt{\sinh ^{2} 2 \rho-\sinh ^{2} 2 \rho_{\min }}, \\
S^{(2)} & =\sqrt{\lambda} \int_{\rho_{\min }}^{\infty} \frac{d \rho}{\cosh \rho} \frac{\sinh ^{2} 2 \rho_{\min }}{\sqrt{\sinh ^{2} 2 \rho-\sinh ^{2} 2 \rho_{\min }}} .
\end{aligned}
$$

For $S^{(1)}$, we further separate it into a divergent part $S_{\text {div }}^{(1)}$ and a finite part $S_{\text {fin }}^{(1)}$

$$
\begin{aligned}
& S_{\mathrm{div}}^{(1)}=\sqrt{\lambda} \int_{\rho_{\text {min }}}^{\rho_{0}} \frac{d \rho}{\cosh \rho} \sinh 2 \rho=2 \sqrt{\lambda}\left(\cosh \rho_{0}-\cosh \rho_{\min }\right), \\
& S_{\text {fin }}^{(1)}=\sqrt{\lambda} \int_{\rho_{\text {min }}}^{\infty} \frac{d \rho}{\cosh \rho}\left(\sqrt{\sinh ^{2} 2 \rho-\sinh ^{2} 2 \rho_{\min }}-\sinh 2 \rho\right) .
\end{aligned}
$$

Here we have introduced the IR cut-off $\rho_{0}$ in $S_{\text {div }}^{(1)}$ as before. Note that $S_{\text {fin }}^{(1)}$ is negative. $S^{(2)}$ in 3.16 is proportional to the function $f\left(\rho_{\min }\right)$ in 3.11$)$

$$
S^{(2)}=\frac{1}{2} \sqrt{\lambda} f\left(\rho_{\min }\right) \sinh 2 \rho_{\min } .
$$

After removing the divergent piece $2 \sqrt{\lambda} \cosh \rho_{0}$ in $S_{\text {div }}^{(1)}$, the regularized action for the annulus worldsheet is found to be 4

$$
S_{\text {reg }}(\text { annulus })=-2 \sqrt{\lambda} \cosh \rho_{\min }+\frac{1}{2} \sqrt{\lambda} f\left(\rho_{\min }\right) \sinh 2 \rho_{\min }+S_{\text {fin }}^{(1)} .
$$

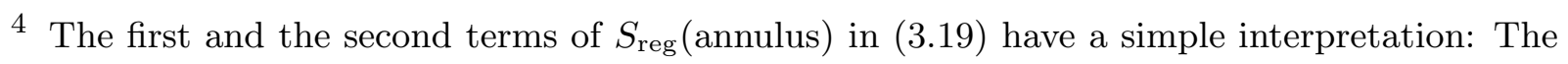
first term is the regularized area of two disks at $\tau= \pm \frac{1}{2} \tau_{0}$, where each disk has a hole of size $\rho_{\text {min }}$ at its center. The second term represents the area of a cylinder $I \times S_{\phi}^{1}$ sitting at $\rho=\rho_{\min }$. Here $I$ denotes the interval of $\tau$ with length $\tau_{0}=f\left(\rho_{\min }\right)$. $S_{\text {fin }}^{(1)}$ represents the correction to this crude approximation that the annulus worldsheet is made of two disks with holes connected by a cylinder of radius $\rho_{\text {min }}$ and length $\tau_{0}$. 
This is written as a function in $\rho_{\text {min }}$, but we would like to study the behavior of this action as a function of $\tau_{0}$ in the region $0<\tau_{0}<\tau_{c}$ where the annulus worldsheet exists. When rewriting this action as a function of $\tau_{0}$, we should be careful about the fact that there are two solutions $\rho_{\min }^{(1)}, \rho_{\min }^{(2)}$ for the equation $\tau_{0}=f\left(\rho_{\min }\right)$ since the function $f\left(\rho_{\min }\right)$ takes a maximum value $\tau_{c}$ at $\rho_{\text {min }}=\rho_{c}$. We assume that $\rho_{\min }^{(1)}<\rho_{c}<\rho_{\min }^{(2)}$. The existence of two solutions for $\tau_{0}=f\left(\rho_{\min }\right)$ implies that given a separation of two loops $\tau_{0}$ there are two annulus configurations corresponding to $\rho_{\min }^{(1,2)}$.

We are interested in the difference $\Delta S$ between the annulus action (3.19) and the action of two disks $2 S_{\text {reg }}($ disk $)=-2 \sqrt{\lambda}$

$$
\Delta S=2 \sqrt{\lambda}\left(1-\cosh \rho_{\text {min }}\right)+\frac{1}{2} \sqrt{\lambda} f\left(\rho_{\min }\right) \sinh 2 \rho_{\text {min }}+S_{\text {fin }}^{(1)} .
$$

As analyzed numerically in [6], the annulus action for the second branch $\rho_{\min }^{(2)}>\rho_{c}$ is smaller than that of the first branch $\rho_{\text {min }}^{(1)}<\rho_{c}$. Therefore, the second branch $\rho_{\min }>\rho_{c}$ gives the dominant contribution to the connected part of correlator $\left\langle W\left(C_{1}\right) W\left(C_{2}\right)\right\rangle_{\text {conn }}$. In particular, $\Delta S$ in (3.20) becomes arbitrarily negative as $\rho_{\min } \rightarrow \infty$, which corresponds to the coincident limit of two loops $\tau_{0} \rightarrow 0$. This can be seen as follows. From (3.14), the large $\rho_{\text {min }}$ limit of $\Delta S$ in $(3.20)$ is written as

$$
\Delta S \sim \sqrt{\lambda}\left[-1+\frac{\sqrt{2 \pi^{3}}}{\Gamma\left(\frac{1}{4}\right)^{2}}\right] e^{\rho_{\text {min }}}+S_{\text {fin }}^{(1)} .
$$

Since the coefficient of $e^{\rho_{\min }}$ is negative and also $S_{\text {fin }}^{(1)}<0, \Delta S \rightarrow-\infty$ as $\rho_{\min } \rightarrow \infty$. As we increase $\tau_{0}$ from $0, \Delta S$ becomes positive at certain value $\tau_{c}^{\prime}$, which occurs before the disappearance of annulus solution, i.e. $\tau_{c}^{\prime}<\tau_{c}$. At $\tau_{0}=\tau_{c}^{\prime}$ the annulus configuration becomes unstable and collapses to two disks $[7]$.

Next we consider the $\rho_{\min }<\rho_{c}$ region. The annulus action for this branch is always larger than that of two disks [6]. We can easily see that the annulus action (3.19) reduces to the action of two disks in the limit $\rho_{\min } \rightarrow 0$

$$
\lim _{\rho_{\min } \rightarrow 0} \Delta S=0
$$

Let us see that $\Delta S>0$ for small $\rho_{\text {min }} \ll 1$. Using (3.14) and $S_{\text {fin }}^{(1)} \sim \sqrt{\lambda} \rho_{\min }^{2} \log \rho_{\text {min }}$ we find

$$
\Delta S \sim-\sqrt{\lambda} \rho_{\min }^{2} \log \rho_{\min }
$$

which is indeed positive. 


\section{Discussion}

In this paper we have considered the minimal surface in the global $A d S_{5}$ which is dual to $1 / 2$ BPS circular Wilson loops. The dual worldsheet is given by the Pincaré disk at a fixed global time. We also revisited the computation of the correlator of two concentric Wilson loops using the global coordinate of $A d S_{5}$. The annulus worldsheet connecting the two loops exists only when the separation $\tau_{0}$ between the two loops is less than some critical value $\tau_{c}$, and it ceases to exist when $\tau_{0}>\tau_{c}$. As argued in [6], this phase transition is different from the point where the annulus and two disks change dominance. It would be nice to understand the physical picture of this transition more clearly.

It would be interesting to study the radial quantization picture of less supersymmetric Wilson loops or other operators such as surface operators. Another interesting direction to study is the commutation relation between a 't Hooft loop and a Wilson loop from the bulk AdS viewpoint. For a circular 't Hooft loop $T(C)$, the dual object is a D1-brane with $A d S_{2}$ worldvolume sitting at a constant global time. In the radial quantization picture, the 't Hooft loop $T(C)$ is represented by an operator $\widehat{T}_{C}(\tau)$ and this operator does not commute with the Wilson loop operator $\widehat{W}_{C}(\tau)[15,16]$

$$
\widehat{T}_{C_{1}}\left(\tau_{1}\right) \widehat{W}_{C_{2}}\left(\tau_{2}\right)=e^{\frac{2 \pi i}{N} \ell\left(C_{1}, C_{2}\right)} \widehat{W}_{C_{2}}\left(\tau_{2}\right) \widehat{T}_{C_{1}}\left(\tau_{1}\right),
$$

where $\ell\left(C_{1}, C_{2}\right)$ is the linking number of two loops inside $S^{3}$. Recently, the correlator of a 't Hooft loop $T\left(C_{1}\right)$ and a Wilson loop $W\left(C_{2}\right)$ in $\mathcal{N}=4 \mathrm{SYM}$ is computed by using the localization of path integral to the two-dimensional Yang-Mills theory on $S^{2}$ [17]. This localization technique works when $C_{1}$ and $C_{2}$ are linked on $S^{3}$. It would be interesting to understand the algebra (4.1) both from the gauge theory side and from the gravity side 0 . On the gravity side, the algebra (4.1) corresponds to exchanging the D1-brane at $\tau=\tau_{1}$ and the fundamental string at $\tau=\tau_{2}$. It would be nice to see how the phase in (4.1) appears in this change of ordering of D1-brane and F1-brane along the $\tau$-direction in global $A d S_{5}$.

\section{Acknowledgment}

This work is supported in part by MEXT Grant-in-Aid for Scientific Research \#19740135.

5 See [18] for some computation of the correlator of concentric circular 't Hooft and Wilson loops on the gravity side. 


\section{References}

[1] J. M. Maldacena, "Wilson loops in large N field theories," Phys. Rev. Lett. 80, 4859 (1998) arXiv:hep-th/9803002.

[2] S. J. Rey and J. T. Yee, "Macroscopic strings as heavy quarks in large N gauge theory and anti-de Sitter supergravity," Eur. Phys. J. C 22, 379 (2001) arXiv:hepth/9803001].

[3] N. Drukker, S. Giombi, R. Ricci and D. Trancanelli, "Supersymmetric Wilson loops on $S^{3}$," JHEP 0805, 017 (2008) arXiv:0711.3226 [hep-th]].

[4] A. Dymarsky, S. S. Gubser, Z. Guralnik and J. M. Maldacena, "Calibrated surfaces and supersymmetric Wilson loops," JHEP 0609, 057 (2006) arXiv:hep-th/0604058].

[5] D. E. Berenstein, R. Corrado, W. Fischler and J. M. Maldacena, "The operator product expansion for Wilson loops and surfaces in the large N limit," Phys. Rev. D 59, 105023 (1999) arXiv:hep-th/9809188.

[6] P. Olesen and K. Zarembo, "Phase transition in Wilson loop correlator from AdS/CFT correspondence," arXiv:hep-th/0009210.

[7] D. J. Gross and H. Ooguri, "Aspects of large N gauge theory dynamics as seen by string theory," Phys. Rev. D 58, 106002 (1998) [arXiv:hep-th/9805129].

[8] A. Sen, "Quantum Entropy Function from $\mathrm{AdS}_{2} / \mathrm{CFT}_{1}$ Correspondence," Int. J. Mod. Phys. A 24, 4225 (2009) arXiv:0809.3304 [hep-th]].

[9] N. Drukker, D. J. Gross and H. Ooguri, "Wilson loops and minimal surfaces," Phys. Rev. D 60, 125006 (1999) arXiv:hep-th/9904191.

[10] J. K. Erickson, G. W. Semenoff and K. Zarembo, "Wilson loops in N = 4 supersymmetric Yang-Mills theory," Nucl. Phys. B 582, 155 (2000) arXiv:hep-th/0003055.

[11] N. Drukker and D. J. Gross, "An exact prediction of N = 4 SUSYM theory for string theory," J. Math. Phys. 42, 2896 (2001) arXiv:hep-th/0010274.

[12] V. Pestun, "Localization of gauge theory on a four-sphere and supersymmetric Wilson loops," arXiv:0712.2824 [hep-th].

[13] K. Zarembo, "Wilson loop correlator in the AdS/CFT correspondence," Phys. Lett. B 459, 527 (1999) arXiv:hep-th/9904149.

[14] H. Kim, D. K. Park, S. Tamarian and H. J. W. Muller-Kirsten, "Gross-Ooguri phase transition at zero and finite temperature: Two circular Wilson loop case," JHEP 0103, 003 (2001) arXiv:hep-th/0101235.

[15] G. 't Hooft, "On The Phase Transition Towards Permanent Quark Confinement," Nucl. Phys. B 138, 1 (1978).

[16] G. 't Hooft, "A Property Of Electric And Magnetic Flux In Nonabelian Gauge Theories," Nucl. Phys. B 153, 141 (1979).

[17] S. Giombi and V. Pestun, "The 1/2 BPS 't Hooft loops in N=4 SYM as instantons in 2d Yang-Mills," arXiv:0909.4272 [hep-th]. 
[18] A. Gorsky, A. Monin and A. V. Zayakin, "Correlator of Wilson and 't Hooft Loops at Strong Coupling in N=4 SYM Theory," Phys. Lett. B 679, 529 (2009) arXiv:0904.3665 [hep-th]]. 